\title{
Generic bifurcation structures of Arnol'd tongues in forced oscillators
}

\author{
Knudsen, Carsten; Sturis, Jeppe; Thomsen, Jesper Skovhus
}

Published in:

Physical Review A

Link to article, DOI:

10.1103/PhysRevA.44.3503

Publication date:

1991

Document Version

Publisher's PDF, also known as Version of record

Link back to DTU Orbit

Citation (APA):

Knudsen, C., Sturis, J., \& Thomsen, J. S. (1991). Generic bifurcation structures of Arnol'd tongues in forced oscillators. Physical Review A, 44(6), 3503-3510. https://doi.org/10.1103/PhysRevA.44.3503

\section{General rights}

Copyright and moral rights for the publications made accessible in the public portal are retained by the authors and/or other copyright owners and it is a condition of accessing publications that users recognise and abide by the legal requirements associated with these rights.

- Users may download and print one copy of any publication from the public portal for the purpose of private study or research.

- You may not further distribute the material or use it for any profit-making activity or commercial gain

- You may freely distribute the URL identifying the publication in the public portal 


\title{
Generic bifurcation structures of Arnol'd tongues in forced oscillators
}

\author{
Carsten Knudsen, Jeppe Sturis, and Jesper Skovhus Thomsen \\ System Dynamics Group, Physics Laboratory III, Technical University of Denmark, DK-2800 Lyngby, Denmark
}

(Received 11 March 1991)

\begin{abstract}
A detailed numerical bifurcation analysis of the forced Brusselator is performed, exposing local and global bifurcation curves that constitute the internal structure of the dominant Arnol'd tongues. The results of our analyses are presented as phase diagrams and one-parameter bifurcation diagrams. Two theorems concerning the existence of global bifurcations near generic codimension-2 bifurcation points are stated and proved. It is argued that the results are generic to a class of periodically forced selfoscillating systems.
\end{abstract}

\section{INTRODUCTION}

Self-oscillating systems are encountered in most branches of science and engineering. Quite often, several oscillating systems interact, and analysis of the overall behavior is therefore of interest. Self-oscillating systems that are forced by an external oscillating signal represent an important class of coupled oscillators. An inherent feature of periodically forced systems is that the frequency and amplitude of the forcing are exogenous and can therefore be controlled and used as bifurcation parameters. A variety of experimental and theoretical studies have been performed to investigate the properties of periodically forced self-oscillating systems. One of the frequently observed phenomena is phase locking of the endogenous oscillation of the system to the exogenous periodical forcing. Phase locking is a typical nonlinear phenomenon, and the regions of entrainment give information about the degree of nonlinearity in the particular system.

As an interesting example, Guevara, Glass, and Shrier [1] have described a series of experiments in which embryonic chick heart cells were subjected to periodic current pulses. They measured the effect of the forcing upon the rhythm of the cells. Among the observed phenomena were phase locking, period doubling, and irregular response. More recently, clinical experiments performed in humans by Sturis et al. [2] have shown that the period of oscillatory insulin secretion can be entrained to an external periodically varying infusion rate of glucose.

The dynamics of periodically forced self-oscillating systems has also attracted significant theoretical attention. Many authors have used so-called phase diagrams to investigate the structures of the phase-locking regions as a function of amplitude and frequency of the periodic forcing. For relatively low amplitudes, the forcing affects the natural oscillation of the system in one of two ways. Either entrainment is observed, or the resulting oscillation is quasiperiodic, i.e., the external and internal oscillations have incommensurate frequencies $[3,4]$. The regions of entrainment in the frequency-amplitude plane are referred to as Arnol'd tongues, and certain generic qualitative and quantitative features have been derived [3-6].
At higher forcing amplitudes, the Arnol'd tongues begin to overlap, and a wealth of bifurcation phenomena can be observed. Feingold et al. [7] have calculated regions of phase-locked, period-doubled, and chaotic solutions for a simple model of an electronic circuit. Mosekilde et al. [8] have made a similar investigation by mapping out the Arnol'd tongues of a model describing a periodically driven semiconductor device, in which the spatially extended nature of the system was taken into account. Cumming and Linsay [9] have constructed phase diagrams by conducting experiments with an electronic relaxation oscillator. Among other things they have attempted to determine the critical line $[4,5]$ at which the Arnol'd tongues begin to overlap.

In 1968 Prigogine and Lefever [10] proposed a theoretical model of a simple chemical oscillating system, the Brusselator. Although the model is not based upon a particular experimental chemical reaction scheme, it contains some general features in the sense that oscillations occur because an equilibrium solution is unstable. A variation of this model, the so-called forced Brusselator, was investigated by $\mathrm{Kai}$ and Tomita [11] in some detail. They calculated some of the regions of entrainment for varying frequency and amplitude of the forcing. Also period doublings and deterministic chaos arising from this period-doubling cascade were considered. Additional analyses of the model focused on overlap and structure of some higher-period Arnol'd tongues [12-14], and revealed various local and global bifurcations [15].

The purpose of the present paper is to extend the analysis of the forced Brusselator by mapping out the bifurcations that occur in the tongues corresponding to operation synchronously with the drive and at twice this period. We focus on these tongues because, within the limits of realistic parameters, they take up most of the parameter plane. In addition, several experimental studies $[1,2]$ use relatively large amplitudes of the forcing. Therefore, a more detailed understanding of the tongue structure at intermediate and high amplitudes is of interest. The transition from phase locking to quasiperiodicity at the edges of the period-1 and -2 Arnol'd tongues was found by Kevrekidis, Schmidt, and Aris [12] to take place in two qualitatively different ways: at lower amplitudes the shift occurs via a saddle-node bifurcation, while 
at higher amplitudes the edges of some tongues changes to a torus bifurcation curve. This indicated a possibility of several bifurcations within each tongue, which was worthy of a more detailed investigation. Some of the bifurcations we map have previously been reported [11-14], but bifurcation curves were not accurately located. Other bifurcations, which we report, do not appear to have been described previously for this particular model. In addition to numerical investigations, we also state and prove two theorems that provide an explanation for the existence of some global bifurcations occurring within the tongues.

\section{MODEL AND METHODS OF ANALYSIS}

The Brusselator has two variables $X$ and $Y$ that represent two chemical intermediates. It is described by the following reactions:

$$
\begin{aligned}
& A \rightarrow X, \\
& B+X \rightarrow Y+D, \\
& 2 X+Y \rightarrow 3 X, \\
& X \rightarrow E .
\end{aligned}
$$

Here, $A, B, D$, and $E$ denote input and output products of constant concentration. For the forced Brusselator, the supply of the chemical $A$ is not constant but contains a periodic component with amplitude $a$. Expressed in terms of coupled differential equations the model reads

$$
\begin{aligned}
& \frac{d X}{d t}=A+X^{2} Y-B X-X+a \cos (\omega t), \\
& \frac{d Y}{d t}=B X-X^{2} Y .
\end{aligned}
$$

Introducing a new time variable $\tau=\omega t$, the equations of motion can be cast into the form

$$
\begin{aligned}
& \frac{d X}{d \tau}=\frac{A+X^{2} Y-B X-X+a \cos (\tau)}{\omega}, \\
& \frac{d Y}{d \tau}=\frac{B X-X^{2} Y}{\omega} .
\end{aligned}
$$

The advantage of writing the equations in this form is that the period of the forcing is always $2 \pi$. This enables us to apply the software package PATH [16] for numerical bifurcation analyses of both stable and unstable phaselocked solutions. In all simulations to be discussed, we have fixed the parameters $A$ and $B$ to be 0.4 and 1.2 , respectively, leading to a frequency of the unforced system of $\omega_{0}=0.3750375$. $\omega$ and $a$ are the bifurcation parameters, allowing immediate comparison with Kai and Tomita [11]. The phase diagram from Kai and Tomita [11] is redrawn in Aronson et al. [14].

To investigate the model, we solve initial value problems in a stroboscopic map defined by $\tau$ being a multiple of $2 \pi$. Stable as well as unstable limit cycles are located by a numerical Newton iteration scheme. Solutions are then followed by varying parameters slowly until a bifurcation is discovered. Once a bifurcation point (e.g., a period-doubling bifurcation) is found, the bifurcation

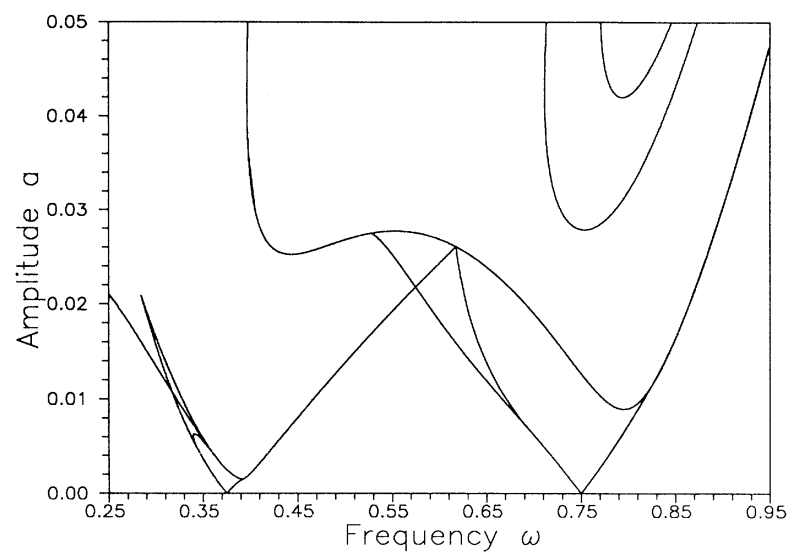

FIG. 1. Overview of bifurcation curves in the frequencyamplitude plane. Comparison with Kai and Tomita [11] is possible. Only the period-1 and -2 tongues are shown. The two curves in the upper-right-hand corner denote period-doubling bifurcations.

curve is traced in the frequency-amplitude plane. These tasks are handled by PATH, and it works well for local bifurcations. Global bifurcations have also been reported in the forced Brusselator [13], and we have developed custom software to study these.

\section{STRUCTURE OF DOMINANT ARNOL'D TONGUES}

Figure 1 shows some of the bifurcation curves in the frequency-amplitude plane. We have left out all tongues with periods larger than 2. For information about these



FIG. 2. Magnification of the region where the period-1 and -2 tongues overlap. The labels indicate the bifurcation type: PD, period doubling; SN, saddle node; $T$, torus; $G$, global bifurcation. The two bullets identify connections of bifurcation curves. 
tongues, we refer to Kai and Tomita [11] and to Aronson et al. [14]. We have encountered period-doubling, saddle-node, torus, and various global bifurcations. Bifurcation curves can either connect or cross. In a point of connection the bifurcation is of codimension 2 . In the present case this occurs when two eigenvalues of the stroboscopic map simultaneously take on the same value of either +1 or -1 . Bifurcations of codimension 2 are marked by bullets in Figs. 2 and 10. When two bifurcation curves cross, as for $(\omega, a) \simeq(0.575,0.0219)$, the bifurcations do not occur for the same solution.

\section{A. Overlap between the period-1 and -2 tongues}

The article by Kai and Tomita [11] shows a phase diagram that indicates that the period- 1 and -2 tongues meet at a certain forcing amplitude. Hereafter they are adjacent, separated by a curve of supercritical perioddoubling bifurcations. We have investigated this region and found that the scenario is somewhat different and more complicated. Figure 2 shows a magnification of the region. In this figure, $\mathrm{PD}, \mathrm{SN}, T$, and $G$ denote perioddoubling, saddle-node, torus, and global bifurcation curves, respectively. As can be seen, the period-1 and -2 tongues meet at a point in the parameter plane (without connecting), and hereafter overlap in a region of increasing values of the forcing amplitude. Subsequently, the left edge of the period-2 tongue, which is a curve of saddle-node bifurcations, meets a curve of perioddoubling bifurcations. This connection represents a codimension- 2 bifurcation point, and it gives rise to a change in stability and direction of the family of period-2 solutions that are born in the period-doubling. The left part of the period-doubling curve acts as a boundary between the period-1 and -2 tongues (see Fig. 1). The upper right edge of the period-1 tongue (a torus bifurcation curve) also joins the period-doubling curve, but in contrast to the scenario suggested by the phase diagram

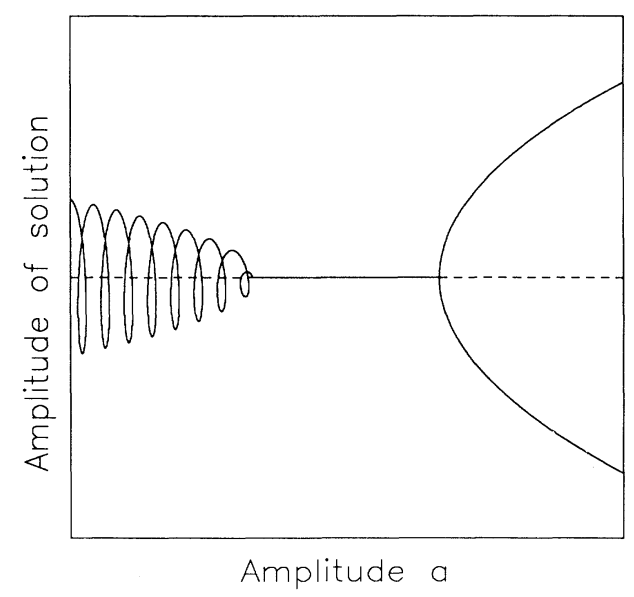

FIG. 3. Principal structure of the bifurcations for increasing forcing amplitude in a region around $\omega=0.5$. Solid curves represent stable solutions, dashed curves are unstable solutions, and spiral-like curves indicate toroidal solutions.

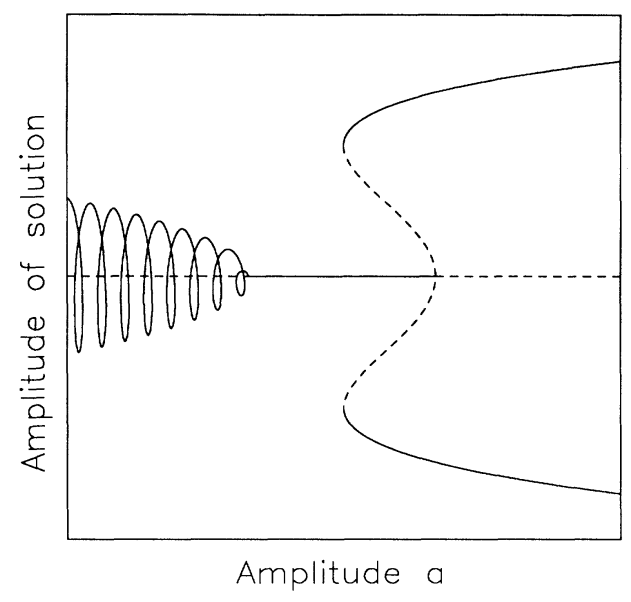

FIG. 4. Principal structure of the bifurcations for increasing forcing amplitude in a region around $\omega=0.55$.

in Kai and Tomita [11], we find that it occurs for a different parameter set. This must be the generic situation, because connections of the two tongue edges to the period-doubling curve at the same point in the parameter plane would represent a codimension-3 bifurcation point, which is degenerate in a two-parameter case. This latter codimension- 2 bifurcation point gives rise to global bifurcations as well, which will be described below.

We have chosen to elucidate the structure of the bifurcations by displaying a series of bifurcation diagrams (Figs. 3-6, 9, and 11) with the forcing amplitude as the parameter. These figures show some of the qualitative features without being accurately drawn to scale. Stable solutions are shown as solid curves, unstable ones as dashed curves, and tori are illustrated as spiral-like curves.

In a region around $\omega=0.5$, the bifurcation scheme is as shown in Fig. 3. For increasing values of the forcing amplitude, the stable solutions lie on a torus that shrinks

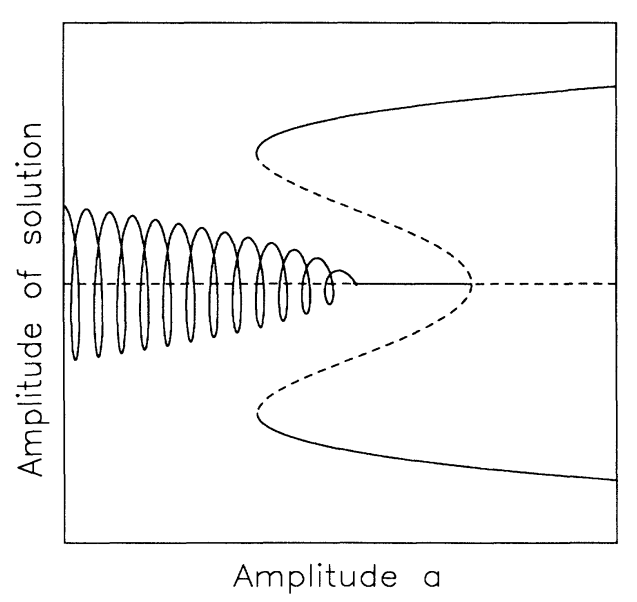

FIG. 5. Principal structure of the bifurcations for increasing forcing amplitude in a region around $\omega=0.6$. 


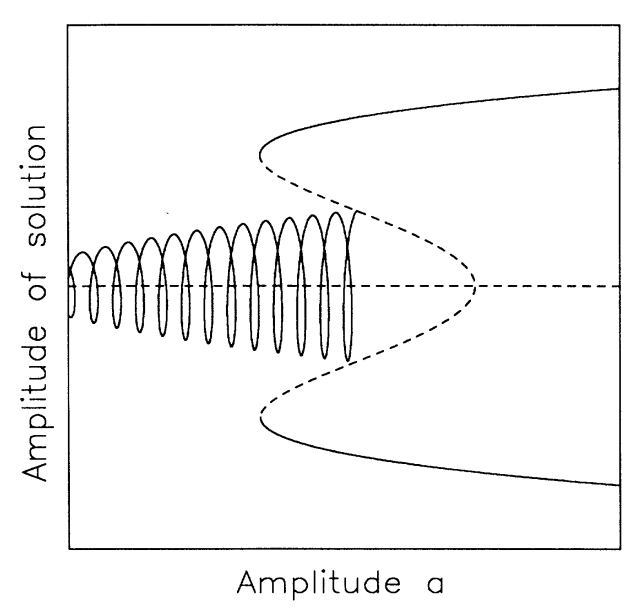

FIG. 6. Principal structure of the bifurcations for increasing forcing amplitude in a region around $\omega=0.64$.

and subsequently bifurcates to a stable period-1 solution in a so-called torus bifurcation. Increasing the amplitude further, the period-1 attractor is destabilized in a supercritical period-doubling bifurcation, i.e., a stable period-2 solution is born and coexists with the unstable period-1 limit cycle.

To the right of $\omega \simeq 0.527$ a qualitative change has occurred. Figure 4 shows the corresponding bifurcation diagram. The torus bifurcation is the same as before, but the period doubling is now subcritical, i.e., an unstable period-2 solution is created and coexists with the stable period-1 solution. The unstable period- 2 solution turns in a saddle-node bifurcation, and thereby becomes stable and coexists with the stable period-1 solution. The inset of the unstable period- 2 saddle is the boundary between the basins of attraction for the stable limit cycles. The qualitative change is due to the connection of the perioddoubling curve and a saddle-node curve, the latter being the left edge of the period-2 tongue. Note that the transition between period-1 and period- 2 phase locking involves a jump in attractor size.

The next bifurcation diagram, shown in Fig: 5, is almost identical to the previous one. The only difference is that the torus bifurcation and the saddle-node bifurcation have exchanged positions with respect to the control parameter. This means that in addition to a region of coexisting period-1 and period- 2 limit cycles, we also have a region of the frequency-amplitude plane in which a stable period-2 limit cycle and a stable torus solution coexist. The basin boundary between the two attractors is still the inset of the unstable period-2 saddle. In the phase diagram, the exchange of location of the torus and saddlenode bifurcations appears as a crossing (not a connection) of the left edge of the period-2 tongue (saddle-node curve), and the right edge of the period-1 tongue (torus curve). The transition from phase locking within the period-2 tongue to a torus solution outside the tongue involves a jump in phase space, because the saddle-node pair does not lie on a torus. This is in contrast to the typical scenario for low forcing amplitudes.
Increasing the frequency beyond 0.62 , we encounter a global bifurcation. Figure 6 illustrates the scenario partially. The torus bifurcation has now disappeared. Following the toroidal solution towards higher amplitudes, the torus vanishes. Before this happens, however, the torus develops an infinite number of folds. Thus, the attractor becomes chaotic. The folds are small compared to the attractor size, and therefore identification of the chaotic attractor is very difficult. We were able to detect chaos by making large magnifications of a stroboscobic map, revealing the folds. As further evidence of chaos, we computed the Lyapunov spectrum [17] $\lambda_{s}$ for $\omega=0.64101$ and $a=0.016$ and found $\lambda_{s}$ $=(0.283,0,-0.195)$. For a forced system one exponent is always zero, and therefore it was not computed. The chaotic attractor disappears in a collision with the inset of the saddle cycle. This bifurcation is known as a chaotic blue-sky catastrophe [18]. In this paper, we do not examine the chaotic transitions more closely. Inside the torus there is an unstable solution with two eigenvalues outside the unit circle in the stroboscopic map. This solution gains stability in one direction through a supercritical period-doubling bifurcation. When comparing Figs. 2, 5, and 6, it appears that the global bifurcation curve ends on the codimension- 2 point. When following the torus bifurcation curve towards the period-doubling curve, it can be observed that the complex conjugated eigenvalues on the unit circle approach and become -1 on the period-doubling curve.

This leads us to state the following theorem:

Theorem 1. Let $\gamma$ be a codimension-2 bifurcation point in an $n$-dimensional flow, $n>2$, where all other eigenvalues of the Poincare map lie within the unit circle. If a curve of stable torus bifurcations $T$ connects to a curve of period-doubling bifurcations PD in $\gamma$, and the perioddoubled solutions are unstable and point in the direction of the curve of torus bifurcations, then a set $\Gamma$ of global bifurcation points exists between the side of the torus bifurcation curve on which the stable tori exist and the period-doubling bifurcation curve. Furthermore, $\gamma$ is a limit point for $\Gamma$.

Figures 7 and $8(a)$ and $8(b)$ show the scenario described in Theorem 1.

Proof of Theorem 1. Because two eigenvalues lie on and the rest lie within the unit circle, the center manifold theorem [15] guarantees that a two-dimensional invariant and attracting center manifold exists. Thus it suffices to consider the two-dimensional case when the parameters are close to $\gamma$. Consider a curve $\Sigma$ connecting a point $\alpha$ on PD to point $\beta$ on $T$. Suppose that no bifurcations occur along $\Sigma$. This implies that the stable torus must coexist with the period-doubled solutions when these are born at $\alpha$ [Fig. 8(a)]. The unstable periodic solution within the torus is the period-1 solution from the perioddoubling bifurcation, since the bifurcation curves $T$ and PD meet in $\gamma$. The torus must therefore lie outside the unstable period-doubled solutions when these are born. The unstable period-doubled solutions lie outside the bifurcating tori on $T$ at $\beta$ [Fig. 8(b)]. It follows that the unstable period-doubled solutions must intersect the stable torus at some point on $\Sigma$. This is a contradiction, since it 


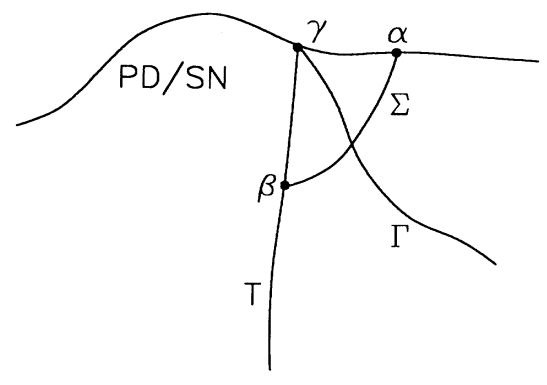

FIG. 7. Principal structure of the situation described in Theorems 1 and 2. PD/SN denotes a period-doubling bifurcation curve in the case of Theorem 1, and a saddle-node bifurcation curve for Theorem 2. $T$ denotes a torus bifurcation curve; $\gamma$ is the codimension- 2 bifurcation point; $\alpha$ and $\beta$ are points on $\mathrm{PD} / \mathrm{SN}$ and $T$, respectively; $\Sigma$ is a curve connecting $\alpha$ to $\beta$; for simplicity, the set $\Gamma$ of global bifurcation points are illustrated as a curve.

implies the existence of at least one bifurcation point on $\Sigma$. Since $\alpha, \beta$, and $\Sigma$ can be chosen arbitrarily close to $\gamma$, it follows that $\gamma$ is a limit point for $\Gamma$, and since $\gamma$ is a codimension-2 bifurcation point, $\Gamma$ must be a set of global bifurcation points, Q.E.D.

Let us make a few comments regarding the nature of the set $\Gamma$ of global bifurcation points. Since the intersection of the curve $\Sigma$ and $\Gamma$ is nonempty, the measure of $\Gamma$ must be, at the least, that of a one-dimensional curve. However, Theorem 1 does not guarantee that $\Gamma$ is a curve, but we strongly suspect that in general $\Gamma$ contains several curves.

To illustrate the theorem, we performed numerical investigations of the forced Brusselator that showed that

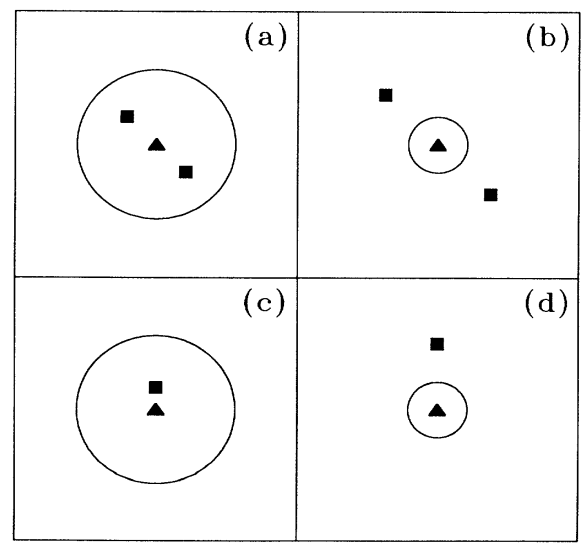

FIG. 8. Hypothetical locations of the torus (circles) and the periodic solutions. The case described in the proof of Theorem 1: (a) near $\alpha$ and (b) near $\beta$. In these two panels a triangle denotes an unstable node and squares represent an unstable period-doubled solution. The case described in the proof of Theorem 2: (c) near $\alpha$ and (d) near $\beta$. In these two panels a triangle denotes an unstable node and a square represents a saddle cycle solution. the torus solutions indeed disappear before the perioddoubling curve, giving rise to at least one curve of global bifurcations, as shown in Figs 1 and 2. We found that the disappearance of the torus solution involves (i) breakdown of the torus to a chaotic attractor, and (ii) disappearance of the chaotic attractor in a blue-sky catastrophe. An alternative scenario could be a homoclinic bifurcation [15] in which the inset of the saddle collides with the torus. Both scenarios leave us with an infinity of intersections between the stable and unstable manifolds of the saddle cycle, so-called horseshoes $[15,18,17]$. The existence of horseshoes implies arbitrarily long (chaotic) transients. We conjecture that the horseshoes must disappear in yet another global bifurcation, because otherwise they would exist for infinitely low forcing amplitudes. In the present case, it thus appears that the set $\Gamma$ of global bifurcation points contains at least three different curves of global bifurcations.

As the frequency is increased even further, the global bifurcation curve(s) and the left edge of the period-2 tongue approach. When the curves join, the transition from phase locking to quasiperiodicity occurs without a jump in phase space. Beyond this point in the parameter plane, the transition for the period-2 tongue is as below the critical line in the circle map [4]. The bifurcation scheme is now as shown in Fig. 9. The exact location of the meeting point has not been determined due to the tangential approach of the curves.

Theorem 1 shows that the existence of global bifurcations is generic for systems in which a codimension-2 bifurcation point exists involving the connection of a torus and a period-doubling curve. Bifurcation analysis of the so-called continuous stirred tank reactor (CSTR) model has been carried out by Kevrekidis, Aris, and Schmidt $[19,20]$, and they have reported that such a codimension2 bifurcation point exists, however, they did not associate global bifurcations with this point. We performed a numerical study of the forced CSTR model in a region near the codimension- 2 bifurcation point, and found evidence

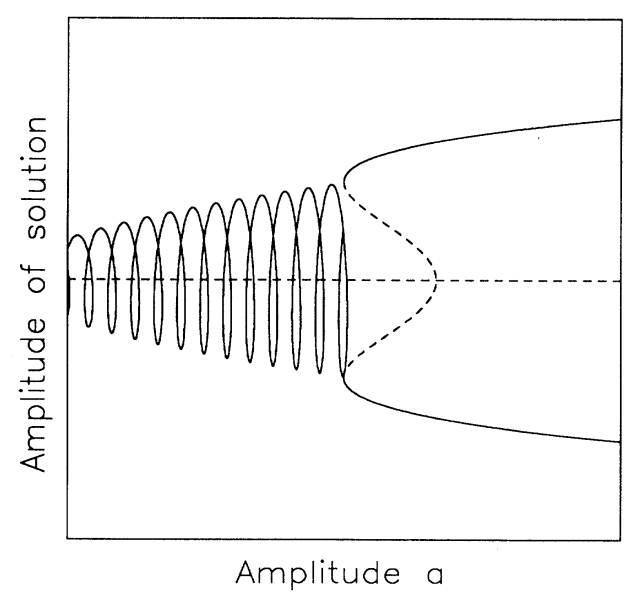

FIG. 9. Principal structure of the bifurcations for increasing forcing amplitude in a region around $\omega=0.74$. 
of a curve of global bifurcations. Chaotic solutions were also found.

\section{B. Bifurcations within the period-1 tongue}

A feature of the period-1 tongue is that at lower forcing amplitudes the transition from phase locking to quaisperiodicity occurs via a saddle-node bifurcation, while at higher amplitudes a torus bifurcation is responsible for the loss of entrainment. It can be seen in Fig. 10 that several bifurcation curves exist. The lower-left saddle-node edge of the tongue crosses (without connecting) the torus curve, and at $(\omega, a) \simeq(0.285,0.021)$ it makes a sharp turn and almost heads in the opposite direction. Subsequently it connects to the torus curve that constitutes the left-hand side of the tongue at higher amplitudes. This codimension-2 bifurcation point is characterized by having two eigenvalues of +1 . Before the connection, one eigenvalue of the saddle-node bifurcation lies within the unit circle. Passing through the connection point, this eigenvalue crosses the unit circle transversely. At this point, the saddle-node curve therefore becomes unstable, continues, and later turns sharply and connects to the torus curve that forms the right edge of the tongue at higher amplitudes. After the connection, the node of the saddle-node pair is again stable, and represents the lower-right edge of the tongue. The existence of an unstable saddle-node bifurcation curves was noted by Kevrekidis, Schmidt, and Aris [12]. In addition to these local bifurcations, we also identified global bifurcations within the period-1 tongue. The presence of global bifurcations has previously been identified by Kevrekidis [13] as homoclinic bifurcations.

This leads us to state the following theorem.

Theorem 2. Let $\gamma$ be a codimension-2 bifurcation point

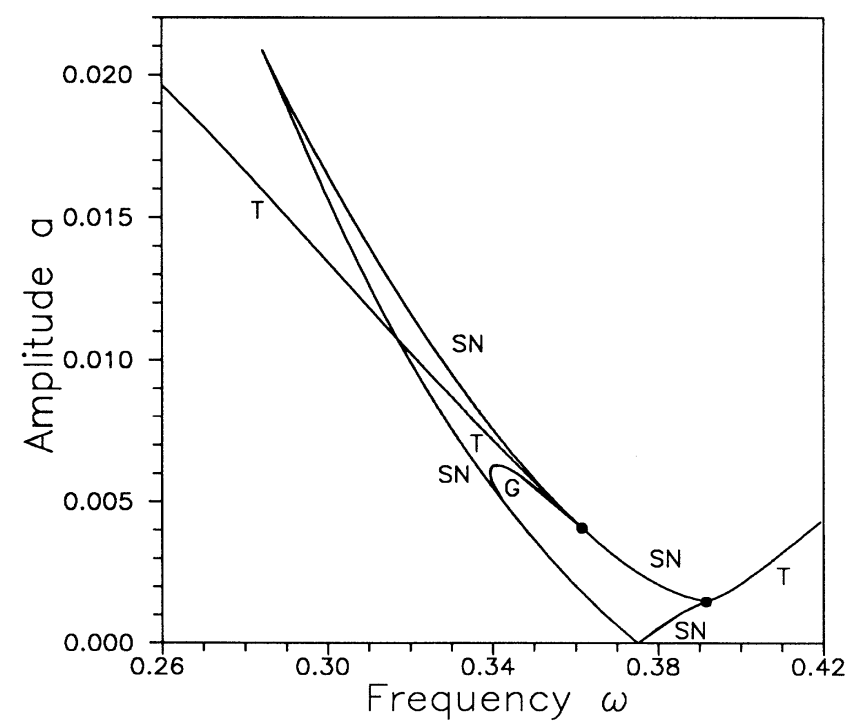

FIG. 10. Magnification of the lower part of the period-1 tongue. The labels indicate the bifurcation type: $\mathrm{PD}$, period doubling; SN, saddle node; $T$, torus; $G$, global bifurcation. The two bullets identify connections of bifurcation curves. in an $n$-dimensional flow, $n>2$, where all other eigenvalues of the Poincaré map lie within the unit circle. If a curve of stable torus bifurcations $T$ connects to a curve of saddle-node bifurcations $\mathbf{S N}$ in $\gamma$, and the node of the saddle-node pair is unstable and points in the direction of the curve of torus bifurcations, then a set $\Gamma$ of global bifurcation points exists between the side of the torus bifurcation curve on which the stable tori exist and the saddle-node bifurcation curve. Furthermore, $\gamma$ is a limit point for $\Gamma$.

Figures 7 and 8(c) and 8(d) show the scenario described in Theorem 2.

Proof of Theorem 2. Because two eigenvalues lie on and the rest lie within the unit circle, the center manifold theorem [15] guarantees that a two-dimensional invariant and attracting center manifold exists. Thus it suffices to consider the two-dimensional case when the parameters are close to $\gamma$. Consider a curve $\Sigma$ connecting a point $\alpha$ on $\mathrm{SN}$ to a point $\beta$ on $T$. Suppose that no bifurcations occur along $\Sigma$. This implies that the stable torus must coexist with the saddle-node pair when this is born at $\alpha$ [Fig. 8(c)]. The unstable periodic solution within the torus is the node from the saddle-node bifurcation, since the bifurcation curves $T$ and SN meet in $\gamma$. The torus must therefore lie outside the unstable saddle-node pair when this is born. The saddle solution lies outside the bifurcating tori on $T$ at $\beta$ [Fig. 8(d)]. It follows that the saddle cycle must intersect the stable torus at some point on $\Sigma$. This is a contradiction, since it implies the existence of at least one bifurcation point on $\Sigma$. Since $\alpha, \beta$, and $\Sigma$ can be chosen arbitrarily close to $\gamma$, it follows that $\gamma$ is a limit point for $\Gamma$, and since $\gamma$ is a codimension-2 bifurcation point, $\Gamma$ must be a set of global bifurcation points. Q.E.D. The comments to Theorem 1 apply to Theorem 2 as well.

For $\omega=0.34$ the bifurcation diagram is as shown in Fig. 11. For increasing values of $a$, the stable toroidal solution coexists with the stable period 1 , which is born in

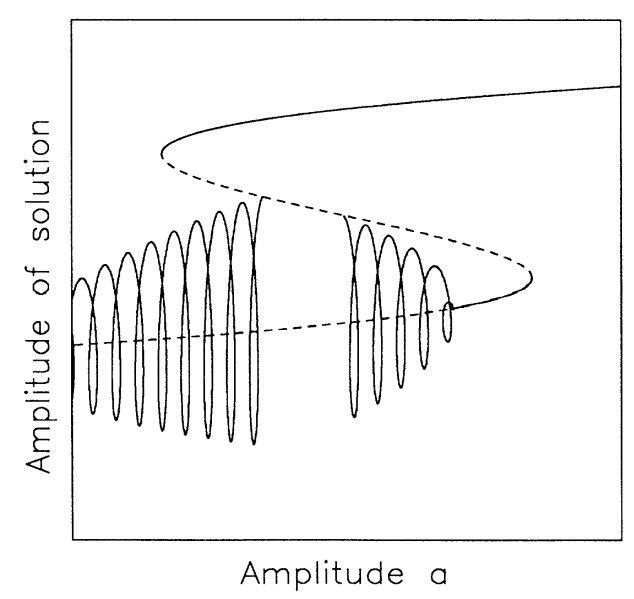

FIG. 11. Principal structure of the bifurcations for increasing forcing amplitude in a region around $\omega=0.34$. Solid curves represent stable solutions, dashed curves are unstable solutions, and spiral-like curves indicate toroidal solutions. 
a saddle-node bifurcation. For $a \simeq 0.0056905$, the toroidal solution has become chaotic and disappears in a blue-sky catastrophe, involving the inset of the unstable saddle solution that was born in the saddle-node bifurcation. Now the stable period 1 is a global attractor. For $a \simeq 0.00625785$, the bifurcation sequence is reversed, and again, there are coexisting stable period-1 and toroidal solutions. The torus subsequently disappears in a torus bifurcation, in which the unstable period-1 solution inside the torus gains stability. Now two coexisting stable period-1 solutions are present within the period-1 tongue. Finally the stable period-1 solution from the torus bifurcation disappears in a saddle-node bifurcation, and again, there is only one stable solution that is globally attracting.

The codimension- 2 bifurcation point near the right edge of the period-1 tongue may also involve global bifurcations. If the torus curve connects to the saddle-node curve after crossing the unstable part of the saddle-node curve, Theorem 2 applies, and global bifurcations are present. Due to the fact that the torus and the saddlenode curves join very near the point where the saddlenode curve turns, we have not attempted to accurately identify the way in which these two curves join.

\section{DISCUSSION}

We have provided a detailed description of some bifurcation structures in the period- 1 and -2 tongues in the forced Brusselator model. Our analyses provide coherent pictures of the organization of (i) the overlapping region between the period- 1 and -2 tongues, and (ii) the bifurcation curves constituting the period-1 tongues's edges at lower forcing amplitudes. In addition to the numerical investigations we have stated and proved two theorems concerning the existence of global bifurcations near generic codimension- 2 bifurcation points that arise when a curve of stable torus bifurcations connects to either a curve of period-doubling bifurcations or a curve of saddle-node bifurcations.

In Kai and Tomita [11], a triple curve connection was displayed between the right-hand edge of the period-1 tongue, the left-hand edge of the period-2 tongue, and a curve of period-doubling bifurcations. We have shown that the right period-1 edge is a torus curve [12,14], and that the left period-2 edge is a saddle-node edge [12]. Such two curves simultaneously connecting to a perioddoubling curve is not a generic situation in a twoparameter plane, because it would require three different local bifurcations to take place for the same parameter combination. As we have shown, the saddle-node curve and the torus curve do indeed connect to the perioddoubling curve, but this happens for different parameter combinations. At the point where the saddle-node curve connects to the period-doubling curve, the latter changes its type from being supercritical for lower frequencies to subcritical for higher frequencies. The connection between the torus and the period-doubling curves gives rise to global bifurcations as demonstrated.

We believe that the overlap between the period-1 and period-2 tongues is generic for a class of forced oscillating systems. If the period-1 and -2 tongues are adjacent for higher amplitudes, the separator must be a curve of supercritical period-doubling bifurcations. This separator represents both the left edge of the period-2 tongue and the right edge of the period-1 tongue. For low amplitudes these edges are separate, and as we have argued in the text, they cannot generically connect at one point in a situation with only two control parameters. The connection of the curves can be imagined to occur in two different ways: (i) in the manner as shown in Fig. 2, or (ii) the right period-1 edge could intersect the perioddoubling curve to the left of the connection point between the period-doubling curve and the left edge of the period-2 tongue. The case (ii) would not contain an overlapping region. Between the two edges a curve $\Omega$ of period doublings would then be present. Assuming that no other bifurcations take place near the codimension-2 bifurcation point, the following reasoning applies: If the right-edge curve were a saddle-node curve, the period-1 solution from the period-1 tongue would disappear in the saddle-node bifurcation, and subsequently there would be no stable period-1 solutions below $\Omega$ which could perioddouble. If the right edge were a torus curve, the period-1 solution from the period-1 tongue would destabilize in the torus bifurcation, and therefore no stable period-1 solutions would exist below $\Omega$. In principle, in both cases, one could imagine the existence of an additional bifurcation curve of the same type located between the right-edge curve and $\Omega$ and connecting to the codimension-2 bifurcation point. However, from a physical point of view, this would be an unlikely situation, at least for low-dimensional systems.

The scenario in the period-1 tongue also seems to occur in the forced CSTR model, which we take as an indication of genericity. We plan to investigate other models to support this hypothesis.

The right-hand side of the period- 2 tongue (see Fig. 1) was also examined. Here a transition from saddle-node to torus bifurcations was also observed. The unstable supercritical period-doubling curve comes very close to the saddle-node and the torus curves. The bifurcation curves are located within a very small region of the parameter plane, similar to what was found for the forced CSTR model $[19,20]$.

Much of the theoretical investigation of periodically forced oscillators has focused upon one-dimensional circle maps. The results derived from such iterated maps may in many cases be extrapolated to more complicated theoretical or experimental systems involving periodically forced oscillators. However, saddle-node and perioddoubling bifurcations are the only local bifurcations that can occur in one-dimensional circle maps. Both theorems in the present paper concerning existence of global bifurcations involve torus bifurcations, and consequently do not apply to one-dimensional circle maps.

In the case of the periodically forced Brusselator, the transition from phase locking to quasiperiodicity can occur either via a saddle-node bifurcation (phase locking $\rightarrow$ quasiperiodicity on a large torus) or through a torus bifurcation (phase locking $\rightarrow$ quasiperiodicity on a small torus). These two different transitions can be 
differentiated when mathematical models are investigated and they may also be distinguishable in experiments under laboratory conditions. If a torus bifurcation occurs in a particular system, results from circle maps cannot necessarily be applied. On the other hand, if torus bifurcations are observed for some parameter combinations while period-doubling and/or saddle-node bifurcations are seen for other parameter sets, the possibility exists that the two theorems in the present paper are applicable.

\section{ACKNOWLEDGMENTS}

We would like to thank E. Mosekilde and M. Brøns for valuable discussions and good advice.
[1] M. R. Guevara, L. Glass, and A. Shrier, Science 214, 1350 (1981).

[2] J. Sturis, E. Van Cauter, J. D. Blackman, and K. S. Polonsky, J. Clin. Invest. 87, 439 (1991).

[3] V. I. Arnol'd, Am. Math. Soc. Trans. 46, 213 (1965).

[4] M. H. Jensen, P. Bak, and T. Bohr, Phys. Rev. A 30, 1960 (1984).

[5] T. Bohr, P. Bak, and M. H. Jensen, Phys. Rev. A 30, 1970 (1984).

[6] R. E. Ecke, J. D. Farmer, and D. K. Umberger, Nonlinearity 2, 175 (1989).

[7] M. Feingold, D. L. Gonzales, O. Piro, and H. Vitturo, Phys. Rev. A 37, 4060 (1988).

[8] E. Mosekilde, R. Feldberg, C. Knudsen, and $\mathbf{M}$. Hindsholm, Phys. Rev. B 41, 2298 (1990).

[9] A. Cumming and P. S. Linsay, Phys. Rev. Lett. 59, 1633 (1987).

[10] I. Prigogine and R. Lefever, J. Chem. Phys. 48, 1695 (1968).
[11] T. Kai and K. Tomita, Prog. Theor. Phys. 61, 54 (1979).

[12] I. G. Kevrekidis, L. D. Schmidt, and R. Aris, Chem. Eng. Sci. 41, 1263 (1986).

[13] I. G. Kevrekidis, AIChE J. 33, 1850 (1987).

[14] D. G. Aronson, R. P. McGehee, I. G. Kevrekidis, and R. Aris, Phys. Rev. A 33, 2190 (1986).

[15] J. Guckenheimer and P. Holmes, Nonlinear Oscillations, Dynamical Systems, and Bifurcations of Vector Fields (Springer-Verlag, New York, 1983).

[16] C. Kaas-Petersen, PATH-User's Guide (University of Leeds, Leeds, UK, 1987).

[17] A. J. Lichtenberg and M. A. Lieberman, Regular and Stochastic Motion (Springer-Verlag, New York, 1983).

[18] J. M. T. Thompson and H. B. Stewart, Nonlinear Dynamics and Chaos (Wiley, New York, 1986).

[19] I. G. Kevrekidis, R. Aris, and L. D. Schmidt, Physica 23D, 391 (1986).

[20] I. G. Kevrekidis, R. Aris, and L. D. Schmidt, Chem. Eng. Sci. 41, 1549 (1986). 